\title{
Differences in manufacturing traditions and assemblage-level patterns: The origins of cultural differences in archaeological data
}

\author{
Kerstin Schillinger ${ }^{1}$, Alex Mesoudi ${ }^{2}$, and Stephen J. Lycett ${ }^{1 *}$ \\ ${ }^{1}$ Department of Anthropology, University at Buffalo, The State University of New York (SUNY), 380 \\ MFAC-Ellicott Complex, Amherst, New York 14261, USA \\ ${ }^{2}$ Human Biological and Cultural Evolution Group, Department of Biosciences, College of Life and \\ Environmental Sciences, University of Exeter, Penryn Campus, Cornwall, TR10 9FE, UK \\ *Corresponding author: sjlycett@buffalo.edu
}

\begin{abstract}
A relationship between behavioral variability and artifactual variability is a founding principle of archaeology. However, this relationship is surprisingly not well studied empirically from a "microevolutionary" perspective. Here, we experimentally simulated artifactual variation in two populations of "artifact" manufacturers, involving only a single behavioral difference in terms of their "tradition" of manufacturing tool. We then statistically analyzed shape variation in the resultant artifacts. In many respects, patterned differences might not have been expected to emerge given the simple nature of the task, the fact that only a single behavioral variable differed in our two populations, and all participants copied the same target artifact. However, multivariate analyses identified significant differences between the two "assemblages." These results have several implications for our understanding and theoretical conceptualization of the relationship between behavior and artifactual variability, including the analytical potency of conceiving of artifacts as the product of behavioral "recipes" comprised of individual "ingredient" behavioral properties. Indeed, quite trivial behavioral differences, in generating microevolutionarily potent variability, can thus have long-term consequences for artifactual changes measured over time and space. Moreover, measurable "cultural" differences in artifacts can emerge not necessarily only because of a strict "mental template," but as the result of subtle differences in behavioral "ingredients" that are socially learned at the community level.
\end{abstract}


Archaeology may succinctly be defined as "the study of relationships between human behavior and material culture" (Reid et al. 1975:864). It has long been recognized that such an emphasis inevitably places study of temporal and spatial changes in the form and variation of artifacts at the center of archaeological endeavor (Clarke 1968). Hence, although a diverse array of theoretical perspectives on artifact variability certainly exist within the discipline, consideration of the character (i.e., form) and variation over time and space of artifacts and their attributes is the quintessential manner in which archaeologists infer behavioral features of the past (Schiffer and Skibo 1997).

Given these factors, a basic assumption in archaeology is that measureable differences between artifacts and assemblages (over either time or space) reflect behavioral differences. For instance, Schiffer and Skibo (1997:28) assert that "formal variability is caused, in a proximate fashion, by artisans executing different sequences of material procurement and manufacture activities" during the production of artifacts. This premise is reinforced further by recognition that artifacts are the product of "behavioral recipes" (e.g., Krause 1985; Schiffer and Skibo 1987). Such behavioral recipes consist of the specific tools and facilities employed during manufacture, the procedures, actions and skills deployed to achieve suitable outcomes, and/or rules applied to solve specific problems that might arise (Schiffer and Skibo 1987:597). Accordingly, it is recognized that individuals and communities may alter, change, and otherwise manipulate these recipes in order that specific outcomes are achieved, or in strategic response to any manner of "functional" feedback, broadly defined (Bleed 1986, 2001; Meltzer 1981; Schiffer and Skibo 1987, 1997; Skibo and Schiffer 2001).

Cultural evolutionary approaches to archaeological data further emphasize that such recipes may be transmitted cross-generationally via social learning (e.g., Eerkens and Lipo 2007; Jordan 2015; Lycett 2015; O'Brien et al. 1994, 2010; VanPool et al. 2008). Social learning may be defined broadly as "learning that is influenced by observation of, or interaction with, another animal (typically a conspecific) or its products" (Heyes 1994: 207), which actually means that a variety of distinct mechanisms can be used in the learning of "behavior recipes" used in artifact production (Lycett 2015). Moreover, cultural evolutionary approaches to archaeological data have also emphasized that incipient differences between alternative forms of behavior, and ultimately alternative forms of artifacts, might therefore be subject to further evolutionary forces that 
exaggerate differences between artifactual assemblages produced by different populations of people over time or in different places (Eerkens and Lipo 2007; Lycett 2015; O'Brien and Lyman 2000; Shennan 2000). In the context of cultural transmission systems ("traditions"), such evolutionary forces could take the form of either selective biases (e.g., choosing to produce one pot form over another) or random factors that result in cultural "drift" in the form and variation of artifactual products (Eerkens and Lipo 2005, 2007; Hamilton and Buchanan 2009; Lycett 2008; Lyman et al. 2009; Mesoudi and O'Brien 2008; Mesoudi 2011; Neiman 1995; O’Brien and Lyman 2000; Rogers et al. 2009; Shennan 2000, 2011). Again, however, at the crux of such a framework is the idea that subtle variations in artifacts caused by subtle variations in behavior might subsequently become exaggerated by these evolutionary forces (Lycett and von Cramon-Taubadel 2015; VanPool 2001). If artifactual variation is only weakly tied to behavioral variation, then such forces cannot act to exaggerate assemblage-level differences over time and space, no matter how strong they might be. Artifactual variation per se is not enough - that variation has to have a behavioral basis. In other words, the source of such variation has to be transmittable (i.e., culturally "learnable") by means of social interaction ("social learning") if it is to be of relevance in cultural evolutionary systems and subsequently have any implication for inferring behavioral factors via measurement of artifactual variation within such a framework (Lycett and von Cramon-Taubadel, 2015; Lycett 2016; Lycett et al. 2016).

Figure 1 schematically illustrates the aforementioned concept that "recipes" (sensu Schiffer and Skibo 1987) comprised of behavioral "ingredients" underlie formal variability in the traits of artifacts, using pottery forms as an illustrative, hypothetical example. In the case of pottery attributes such as rim shape, neck shape, or handle shape (Figure 1) pertinent behavioral "input" ingredients potentially include whether the pot is hand- or wheel-thrown, the method of tempering, differences in the mechanics of firing, choice of which tools (if any) are used in shaping, and so forth (e.g., Orton et al. 1993; Shepard 1965). Figure 1 also illustrates that raw material properties might act as an "environmental" source of variation in addition to that caused by the behavioral "input" variables that are applied to that raw material during manufacture. To be clear: within this framework, "behavior" is, therefore, the product of any choice or action implemented by human actors that influences details of the recipe involved in artifact production (e.g., choosing to use one type of manufacturing tool over another). When such behavioral features are transmitted via social 
learning, they become cultural traditions of artifact manufacture. Environmental factors, on the other hand, are any additional variables that influence artifactual form that cannot be (or are not) directly changed by human behavior (e.g., inherent raw material properties).

Figure 1 also emphasizes, however, that each behavioral variable might, in principle, simultaneously affect the form of several different artifactual "traits." That is, the effect of a single behavioral "input" variable can potentially be "pleiotropic" (i.e., have many influences) on artifactual form (Lycett 2016). Moreover, Figure 1 also illustrates how individual artifactual traits can also, in principle, be influenced simultaneously by the combined effects of several different behavioral input variables. Hence, many individual artifactual traits are "polygenic" or "multifactorial" in character; i.e., their genesis and ultimate form is influenced by multiple, independent behavioral ingredients (Lycett and von Cramon-Taubadel 2015). Importantly, ethnoarchaeological work in recent decades has empirically highlighted the diverse number of possible behavioral (ingredient) steps and the array of differences that might exist among the same steps in the recipes of different communities, thus further illuminating the complexity and diversity of behavioral recipes involved in the production of hand-crafted artifacts (e.g., Hampton, 1999; Mahius 1993; Rice, 1987; Schiffer and Skibo 1997; Skibo and Schiffer 1995; Wallaert 2008; Wendrich 1999).

Of course, archaeologists cannot observe (or even necessarily infer) all of the separate behavioral components of the "recipes" that led to visible archaeological traditions. When we identify differences between sets of artifacts that might only be visible on a statistical basis, are we justified in thinking that even relatively subtle behavioral differences could potentially underpin such patterns? Such a question would seem to go to the crux of archaeology as "the study of relationships between human behavior and material culture" (Reid et al. 1975:864). The concept of recipes implies that subtle differences at the "ingredient" level, rather than wholesale ones, might impact patterns of artifactual trait variability in statistically detectable ways. This is especially the case in cultural evolutionary approaches where it is suggested that quite subtle differences in artifactual form become the source of variation upon which drift and selective biases operate and so exaggerate out over time to eventually form more overt distinctions between sets of artifacts (Eerkens and Lipo 2005, 2007; Eren et al. 2015; Lycett 2015; O’Brien and Lyman 
2000; Smallwood 2012; VanPool 2001). In other words, according to this scheme, relatively discrete and subtle (microevolutionary) behavioral factors are the basis upon which larger, more obvious (macroevolutionary) spatial and temporal trends in the archaeological record are built. This notion of "population thinking"- describing how individual-level processes gradually alter population-level variation - has been described as one of Darwin's major contributions to biology (Ghiselin 1969), and proved hugely superior to pre-Darwinian "essentialist" or typological schemes in which within-population variation is ignored.

Links between discrete (microevolutionary) processes and broader-scale (macroevolutionary) patterns are not well studied in archaeology. Part of this may be due to the difficulty of teasing out the potential magnitude of subtle, discrete factors in situations where artifacts are the product of many influences and activities. Returning to Figure 1 illustrates this point. While a behavior such as pottery manufacture might be observed ethnographically, the fact that individual behavioral input variables are pleiotropic in effect and measurable traits are polygenic in character, means that identifying the exact magnitude of just one input variable on subtle variations in artifactual form becomes a major challenge. Even experimentally, when producing an artifact such as a pot, the array of factors that might influence subtle (statistical) variations between attributes of individual artifacts, make it difficult to precisely isolate the role of specific, individual behavioral ingredients as opposed to effects that might be generated via implementation of different recipes entirely. Yet, for the reasons we have outlined, studying whether, in principle, even subtle and proximate behavioral differences (i.e., subtle differences between single input variables) can lead to patterned statistical differences in artifacts (i.e., generate microevolutionarily relevant effects) has profound implications for understanding the origins of what might ultimately be observable at the more macroevolutionary scale of the archaeological record. In sum, studying the potential role and magnitude of isolated microevolutionary dynamics is fundamental to improving our understanding of the proximate basis of long-term and large-scale (macroevolutionary) trends observable archaeologically, but studying such factors in a behavioral and artifactual context presents immense challenges.

Over many decades of study in evolutionary biology, profound progress has been made in the understanding of fundamental biological microevolutionary processes (e.g., the basis of heredity, inheritance of specific characteristics, the proximate causes of phenotypic variation, influences on 
mutation rates, and so forth) via the use of so-called "model organisms" (Bataillon et al. 2013; Futuyma and Bennett 2009; Garland and Rose 2009; Mueller 2009). Model organisms are valuable because they enable a more secure understanding of phenomena of wide interest, from seemingly discrete, even trivial, laboratory experiments. Commonly used model organisms, such as fruit flies (Drosophila spp.), tend to have a variety of characteristics that make them particularly amenable for use in such experiments, including economy, speed of replication, relative simplicity, andmost importantly — controllability and manipulability (e.g., Ashburner et al. 2005; Ashburner and Novitski 1976; Greenspan 2004). The most suitable model organisms thus display some of the complexities of the phenomenon of general interest, yet are not so complex that they are unwieldy in experimental settings and facilitate a more precise study of discrete factors and processes. For paleobiologists interested in understanding the evolution of animals such as Tyrannosaurus rex, experiments looking at subtle differences in fruit flies generated under highly controlled laboratory conditions may seem far removed from the phenomena of interest; and yet, biologists have long recognized the immense value of this body of work in respect to increasing an understanding of specific microevolutionary factors, which are particularly important for building a robust evolutionary theory that can be applied in broader contexts (Bataillon et al. 2013). Elsewhere, we have argued that in regard to the study of cultural evolutionary processes, simple experiments that replicate certain aspects of artifactual form (e.g., their size and/or shape) make a particularly useful subject of study for similar reasons (Lycett et al. 2015; Schillinger et al. 2014a). Hence, similarly to the use of such experiments in evolutionary biology, the examination of "model artifacts" in controlled laboratory settings can help shed light on fundamental microevolutionary process that are directly relevant to issues that must be considered when examining wide-scale and long-term patterns in the archaeological record (Eerkens 2000; Kempe et al. 2012; Lycett et al. 2016; Mesoudi and O’Brien 2008; Schillinger et al. 2014a, 2014b; 2015).

Here, we use a "model artifact" experimental framework to examine the question of whether differences in just a single input "ingredient" of an artifactual recipe might lead to systematic and statistically identifiable differences between different sets of artifacts. We deliberately focus on whether a difference in just a single "input" ingredient variable can lead to statistically identifiable patterns in the shape of artifacts, given that shape is a relevant (and empirically measureable) property of a wide range of artifacts. In order to test the essential strength of the notion that even 
quite trivial differences in behavior might lead to such systematic shape differences in artifacts, we deliberately designed the experiment such that it is weighted against generating systematic patterns in the data. Hence, we used a simple task that did not require a great deal of "skill" or prolonged period of learning to execute, we measured effects after just one "generation" of copying, all participants copied exactly the same target artifact, and the difference in our experimentally manipulated input ("ingredient") behavior was one of degree rather than kind, involving only a switch between two alternative types of shaping implement that generated exactly the same kind of action (i.e., cutting and removal of material). The logic of testing whether such a simple difference in just a single experimental variable could generate statistically significant and immediate effects at the artifactual level, was to help to begin to more specifically address the issue of scale in relation to behavior and extent of "signal" in artifactual data. In other words, can trivial differences (even in principle) generate a statistically significant effect or are more largescale changes required? If small-scale behavioral changes can generate statistically significant patterns in "model artifacts" then this helps strengthen the assumed link that observable variation in archaeological artifacts is caused "in a proximate fashion" (Schiffer and Skibo 1997: 28) by differences in behavioral recipes. Indeed, if a singular, simulated behavioral difference in a laboratory setting can generate statistically significant effects in artifacts under such conditions, then this emphasizes the power of the concept of "recipes" when multiple ingredients are manipulated, as is likely to be the case in reality-i.e., in most archaeological situations.

\section{Materials and Methods}

Two contrasting experimental conditions were employed to examine these issues, requiring participants to engage in a simple copying task. Participants were asked to faithfully copy the shape of a single "target" (model) handaxe from a standardized block of foam using a cutting tool. It should be emphasized that our choice of using handaxe form here was not motivated by concern solely with handaxes (or indeed any other lithic form per se). Rather, as with the use of model organisms such as Drosophila in biology, use of handaxe form provides a balance between factors that make replication of this form experimentally useful in order to provide insights into parameters and processes that have far reaching consequences across a range of artifactual situations. The simple and straightforward nature of the task, requiring no specialized knowledge, 
ameliorates the impact of variation in individual "skill" levels that become pertinent in the case of archaeological artifacts such as real handaxes, pots, baskets, arrowheads, and so forth. The experimental conditions differed, however, in respect to the cutting tool provided to participants in each condition. In one condition, participants used a plastic knife to shape the handaxe, while in the alternative condition, participants were required to use a metallic vegetable-peeler. All other aspects of the experiment were identical across both conditions. Morphometric properties (sizeadjusted shape data) of the artifacts produced in each condition were then subjected to multivariate statistical analysis.

\section{Participants}

A total of 60 participants took part in the experiment. The majority of participants were undergraduate and postgraduate students, recruited via advertisement. We requested basic demographic data from the participants including their sex and age category. Of the participants, 30 were female (aged between 18 to a maximum of 44 years) and 30 were male (aged between 18 to a maximum of 34 years), with age information being based on self-reported category. Informed consent was obtained from all participants and a financial compensation of $£ 4$ ( $\sim$ 6 US) was paid to each volunteer for their participation.

\section{Materials}

We asked participants to copy the shape of a "model artifact" during the experiment. Shape is a component of variation in all three-dimensional artefacts - everything from projectile points, pots, handaxes, and baskets, through to tombstones, statues, house-plans, footballs, and spacecraft. The shape of an artifact (distinct from scale or "size") can potentially be manipulated during design and manufacture, either due to aesthetic preferences, or because one shape performs differently to others; hence, "shape" is a parameter of artifactual variation that may be functionally relevant in a range of techno-functional or socio-functional fields (sensu Schiffer and Skibo 1987).

The experiment required all participants to copy the same three-dimensional foam "target form," modeled after the shape of an Acheulean handaxe (Figure 2). It is generally agreed that the production of "handaxes" during the Palaeolithic represents a shift from the manufacture of 
relatively simple cutting tools (flakes) produced by bouts of knapping that were not necessarily directed toward the shaping of deliberate core forms, to one where manufacturing procedures were deliberately oriented toward shaping the block of stone in a strategic manner (Gowlett 2006; Roche 2005). The effective production of stone handaxes via knapping requires levels of skill and experience that are built over months, if not years, of practice (Edwards 2001). Conversely, foam "handaxes" are easily manufactured, requiring no specialist skills or knowledge. However, this task still requires the manufacturer to accurately copy integrated and multivariate 3D shape properties, such as relative length, breadth, and thickness variables, and overall outline shape, in order to accurately replicate overall artifactual form. Hence, as with model organisms in biology, this task is strategically useful in an experimental context given our wider goals.

The "target" model and all subsequent copies of it produced by the participants were manufactured from standardized blocks of foam. These high-density foam blocks (OASIS DRY SEC foam) are designed to be used by professional florists to securely hold the stems of artificial flowers. The material is robust to handling, but designed to be malleable so that it can be easily cut and modified into desired sizes and shapes using commonplace implements such as knives and scissors. The machine-cut foam blocks were acquired from the manufacturer with standardized dimensions (22.3 $\mathrm{cm}$ in length, $7.8 \mathrm{~cm}$ in thickness, and $11 \mathrm{~cm}$ in width), which allowed for the starting conditions during the experiment to be identically replicated across all of the participants.

\section{Experimental Conditions and Procedure}

Replicas of handaxes were manufactured from the foam blocks using one of two different tools. One foam manipulation tool was a simple plastic table-knife (Figure 3). The second device for foam manipulation was a metallic vegetable-peeler (REX Swiss Quality peeler). As shown in Figure 3, the metallic peeler incorporates a movable blade positioned perpendicular to the handle (blade moves with 90 degrees freedom). In order to deliberately control for handedness, both the plastic knife and the metallic peeler were specifically chosen for equal suitability for both left- and right-hand use; however, as an additional precaution we also ensured that the proportion of right and left handed participants in each condition was statistically identical $\left(\chi^{2}=0.5769, p=0.447\right)$. Participants were provided with a lab coat, mouth protection, and safety-glasses to guard from foam dust. 
All other factors of the manufacturing process remained constant, ensuring that the only factor of manipulation in this experiment was the type of manufacturing tool employed in two separate populations of "handaxe" manufacturers. In the first experimental condition, defined here as the metallic-peeler condition, participants produced handaxe replicas from the standardized foam block by applying the metallic peeler. In the second condition, termed the plastic-knife condition, participants used the plastic knife to produce the foam handaxe replicas.

The participants in this experiment were divided equally between the two experimental conditions such that there were 30 participants in each group. Laboratory tests have shown that human males and females may differ (statistically) in their performance during tasks involving spatial perception and mental rotation of 2D objects (Halpern 2000; Linn and Peterson 1986; Voyer et al. 1995; Wynn et al. 1996), although such sex-related performance effects have also been shown to reduce substantially whenever 3D objects are involved (Robert and Chevrier 2003). However, for the purposes of precaution, potential sex differences were controlled for here by dividing participants into the alternate tool conditions such that there were equal numbers of 15 females and 15 males in each condition.

While participants in both conditions were asked to faithfully copy the overall shape and form of the target foam handaxe, it was clearly specified that the priority goal was to copy the shape of the target form. To enhance motivation levels in the participants, they were informed that the participant who most accurately copied the target foam handaxe would receive a book voucher to the value of $£ 20$, in addition to the $£ 4$ compensation. Prior to commencing the experimental task, every participant read an instruction sheet and then was handed the target foam handaxe. Each participant was then instructed to inspect the target form from all sides and angles for one full minute, although the model remained with the participants throughout the exercise and they were permitted to make direct comparisons with their own copy at any time, which also controlled for potential memory effects (e.g., Eerkens 2000). Once the inspection time was over, each participant was placed at a table and provided with a standardized foam block and one of the two alternative types of manipulation tool. The participants were informed that they had a 20 minute timeframe to complete the manufacturing task. Previous experiments have demonstrated that 20 minutes is sufficient time to effectively complete the replication task (Schillinger et al. 2014b, 2015). 
Participants were provided with a countdown timer and were also reminded verbally of the remaining time left for task completion at five-minute intervals. Participants were also permitted to wear spectacles or contact lenses, where necessary, for close-up tasks to avoid biases resulting from visual disparities between participants. The use of any additional devices that could enhance perceptual accuracy (e.g., scaled-rulers) was not, however, permitted. While each participant had only one attempt at the replication task, all participants managed to complete the manufacturing task effectively within the provided timeframe.

\section{Morphometric analysis and computation of shape data}

A total of 42 morphometric variables were recorded on each artifact replica produced by the participants. Of these, 28 measurements were acquired from the plan-view perspective (Supplemental Figure 1a) and 14 measurements were obtained from the profile-view perspective (Supplemental Figure 1b). The measurements were digitally recorded from photographic images that were imported into the freely-available morphometric software tpsDig v2.16 (Rohlf 2010). Standardized photographic images of the plan- and profile-view perspective were taken using a Fujifilm DSLR camera (30x zoom lens: $24-720 \mathrm{~mm}$ ) that was attached to a copystand. The shape outline of each handaxe's plan-and profile view was enhanced by placing the specimen on a light box. Each artifact replica was positioned according to a standardized orientation protocol to obtain maximally homologous measurements (Supplemental Figure 2). The measurements for each replica were recorded by superimposing a digital grid on each photographic image that defined the 42 bilateral and lateral measurements. The procedure for obtaining morphometric shape data has been fully described in previous experimental settings (see e.g., Schillinger et al. 2014a, 2014b), and a comprehensive description of the orientation protocol can be found in the online supplementary materials.

Although often conflated, size and shape are two distinct quantitative properties of any threedimensional object (Bookstein 1989; Jungers et al. 1995), including artifacts (Lycett 2009). Since this study's main aim was to investigate differences in shape variation in two populations of artifact replicas as the direct result of distinct tool "traditions," the next step of the data analysis required that shape data be extrapolated from the raw (dimensional) measurements, allowing for the removal of potentially confounding size variation (i.e., isometric scaling). To accomplish this, the 
raw measurement data were size-adjusted via the geometric mean method (Jungers et al 1995; Lycett et al. 2006). This method effectively controls for scaling variation between specimens while the preserving the original shape variation of each specimen in the analysis (Falsetti et al. 1993; Jungers et al 1995). The geometric mean for a series of $n$ variables (e.g., $a_{1}$ to $a_{\mathrm{n}}$ ) may be computed as: $\sqrt[n]{a_{1} \times a_{2} \times a_{3} \times \ldots \times a_{n}}$ (Sokal and Rohlf 1995: 43). The method proceeds on a specimen-byspecimen basis, and involves simply dividing each variable of an individual specimen, in turn, by the geometric mean of all the variables for that particular specimen; this procedure is then repeated for all specimens.

\section{Statistical Analysis of Morphometric Shape Data}

In order to test whether the two experimental behavioral conditions translated into the occurrence of systematic shape differences between the two populations of model artifacts, we undertook a series of multivariate and univariate statistical analyses. Firstly, a principal component analysis (PCA) was used to visualize shape variability among the artifacts produced in the two experimental conditions. PCA facilitates the investigation of the main shape variation between the individual foam handaxe replicas on the basis of a hierarchical approach, such that the first principal component (PC1) extracts the major axis of shape variation and subsequent PCs identify successively less of the total variation between individual specimens.

For further quantitative investigation, a $t$-test was used to compare principal component scores from each of the two tool groups on PC1 (i.e., axis accounting for the largest amount of shape variation among the experimental artifacts). If differences in the two experimental behavioral conditions have resulted in the immediate and systematic occurrence of shape differences between the two populations of "model artifacts," then, a statistically significant $(\alpha=0.05)$ difference between the PC scores from each experimental condition should be evident. Since PC1 will not represent all of the shape variability between specimens, a multivariate analysis of variance (MANOVA), was also conducted on the complete set of PC scores to test whether shape variability between the experimental conditions were significantly different when 100 percent of the shape variation was included. When conducting the MANOVA, both Pillai's trace and Wilks' Lambda statistical analyses $(\alpha=0.05)$ were utilized. All statistical analyses were undertaken in PAST v3.08 (Hammer et al. 2001). 


\section{Results}

The PCA analysis determined that the first two PC scores account for more than 50 percent of the total shape variation (53.3 percent) among the 60 handaxe replicas. PC1 was plotted against PC2 (Figure 4), with PC1 accounting for 32.98 percent of total variation and PC2 representing 20.32 percent of the variation. As is visually discernible, there is some clustering taking place in PC1 among the handaxes from the two conditions (Figure 4). A majority of the handaxes from the metallic-peeler condition can be found in the quadrants displaying positive values on PC1, tending toward a more pointed and relatively thinner shape. Conversely, the majority of handaxes in the plastic-knife condition are found in the quadrants displaying negative values on PC1, exhibiting a trend toward a more oval shape outline and a relatively thicker profile (Figure 4). When statistically comparing shape variability between the two tool conditions using the scores from PC1, the $t$-test established a significant difference $(t=-2.154, d f=58 ; p=0.035)$. Thus, when the axis of largest variation was considered, handaxes from the two experimental conditions displayed statistically distinctive patterns of shape.

The MANOVA also demonstrated statistically significant differences in the PC scores from the plastic knife and the metallic peeler condition (Pillai's trace $=0.872 ; F=2.751 ; d f=42,17 ; p=$ 0.013; Wilks' Lambda $=0.128 ; F=2.751 ; d f=42,17 ; p=0.013)$. Hence, the two contrasting manufacturing conditions generated statistically distinct patterns of shape in their respective sample population.

As noted, we controlled for differences between males and females by having an even number of both sexes distributed between our two experimental conditions; hence, sex cannot account for the results between conditions. However, to put our primary result into comparative context, we also ran the same statistical analyses on the data using sex as the grouping variable. Both the $t$-test $(t=$ 0.9638; $d f=58 ; p=0.34$ ) and the MANOVA (Pillai's trace $=0.5596 ; F=0.5142 ; d f=42,17 ; p$ $=0.96$; Wilks' Lambda $=0.4404 ; F=0.5142 ; d f=42,17 ; p=0.96$ ) displayed no statistical differences, thus emphasizing the strength of the primary result in terms of directional (i.e., systematic and bimodal) shape differences between the two tool-group experimental conditions in the PCA. 
In sum, these results demonstrate that differences in the tools employed when producing the model artifacts was sufficient to generate significantly distinct (i.e., systematic) patterns of shape variation in the two groups, even though all other aspects of the experiment were kept consistent.

\section{Discussion}

The notion that behavioral variability underpins artifactual variability is a key assumption in the field of archaeological endeavor. Indeed, ultimately, a relationship between behavioral variability and artifactual variability is the sine qua non of the entire disciple. At certain levels, behavioral differences (especially involving changes in multiple behavioral domains) will obviously lead to profound differences, and so at larger scales of behavioral difference, the relationship between artifact form and behavior may be taken almost as axiomatic. However, the strength of this relationship - especially at relatively small scales of behavioral and resultant artifactual variation-is surprisingly not well studied empirically from an explicitly "microevolutionary" perspective. This is potentially problematic since many frameworks of inference within the field have long proposed that relatively small-scale behavioral differences are of important longer-term consequence in technological and spatio-temporal patterns of change, and ultimately visible in the archaeological record (e.g., Clarke 1968; O’Brien and Lyman 2000; Schiffer and Skibo 1987, 1997).

Here, we experimentally simulated artifactual variation in two separate populations of "artifact" manufacturers, involving only a single behavioral difference in their "tradition" of manufacturing tool. We then statistically analyzed shape variation in the artifactual products of these two experimental populations of artifact manufacturers using morphometric techniques and multivariate methods. We specifically focused on shape variation since this is a quintessential property of artifactual variation that will have been of aesthetic, functional, and/or social consequence for many past communities of artifact producers. In respect to a range of behavioral and raw material factors that might influence artifactual form in ethnographic situations or in the case of archaeological data (Figure 1), our experiment had the advantage that it was able to hold many key variables constant between participants, except for one specific behavioral "ingredient," which differed in the two experimental "populations" of artifact manufacturers. In many respects, 
statistically patterned (i.e., systematic) shape differences between the two artifactual assemblages might not have been expected to emerge under the experimental conditions we implemented: all participants copied exactly the same "target" shape, we examined differences in "first generation" copies, and the task was a relatively simple one (copy a foam handaxe) requiring no specialized knowledge. Perhaps even more importantly, there was a just single experimentally instigated systematic behavioral difference ("choice" of manufacturing tool) between these two populations, whereby the two experimental populations merely used different types of cutting and shaping tool (a plastic knife versus a metallic vegetable-peeler).

Our statistical analyses, however, identified significant, systematic shape differences between the artifacts produced by each experimental population. That is, shape differences between the two experimental artifact assemblages were "bimodal" in pattern to a statistically significant extent. An equivalent analysis using sex of participants as the grouping variable generated no such effect, thus demonstrating the strength of the primary result in terms of systematic, shape differences between the two tool-group experimental conditions in the PCA. What is particularly striking about these results is that these systematic shape differences emerged in the two artifactual assemblages, even though their manufacturers had been motivated toward copying the same "target" form. In other words, the experimentally instigated behavioral differences between the two populations of artifact manufacturers resulted in quantifiable, statistically patterned differences at the artifactual level, despite the fact that all participants had the same "intentions" with respect to the artifact they were copying.

Despite its highly controlled and "artificial" character, the results of this experiment have several implications for our understanding of the relationship between behavior and measureable artifactual patterning in the archaeological record, at both short-term and long-term scales. The first of these, is that the experiment reiterates the potential analytical potency of conceptualizing artifacts as the products of different behavioral "recipes" (sensu Schiffer and Skibo 1987). At its most powerful, this concept would imply that differences between artifacts (e.g., statistical shape differences in the same class of artifacts at two different localities or in different temporal ranges) might be statistically detectable as a result of relatively subtle behavioral differences, rather than only wholesale changes to multiple different "ingredients." The results of this controlled experiment reveal how potentially sensitive artifactual shape data can be to even relatively subtle 
behavioral differences between populations of artifact makers. Indeed, since archaeology has been described as "the study of relationships between human behavior and material culture" (Reid et al. 1975:864), the fact that even relatively subtle differences in behavior of different human populations can be statistically detected in the shape differences of artifacts is encouraging when it comes to assessing the capacity for artifactual shape variation to reveal meaningful behavioral information over varying temporal and spatial scales.

These experimental results also emphasize that subtle behavioral differences, which are then ultimately the proximate source of relatively subtle, but statistically significant, differences between artifactual assemblages, are potentially the source of even greater and more apparent differences between sets of artifacts visible archaeologically. That is, patterned artifactual variation may emerge from quite trivial behavioral differences, and yet that artifactual variation may end up being of high microevolutionary relevance in wider cultural evolutionary systems. In other words, these results highlight that even subtle behavioral differences between artifact producers may be the incipient source of variation that is subsequently exaggerated out over time to become visibly distinct "traditions" (Eerkens and Lipo 2007; Eren et al. 2015; Lycett and von Cramon-Taubadel 2015; O'Brien and Lyman 2000; Shennan 2000; VanPool 2001). This again highlights the fact that even such subtle and initially non-intentional artifactual differences, if they have any "functional" implications with respect to their performance in given roles (Meltzer 1981; Schiffer and Skibo 1987, 1997), can potentially be elevated further over time via wider cultural evolutionary processes (Eerkens and Lipo 2007; Jordan 2015; Lycett 2015; O’Brien et al. 2010; Shennan 2011; VanPool 2001). However, it is also worth reemphasizing that both selective biases and drift (i.e., stochastic processes) will have the capacity to influence and instigate such long-term change in such variations as a direct result of their proximate behavioral basis (Buchanan and Hamilton 2009; Eerkens and Lipo 2007; Hamilton and Buchanan 2009; Lycett 2008; Lycett and von CramonTaubadel 2015; Lyman et al. 2009; Mesoudi 2011; Neiman 1995; Rogers et al. 2009; Shennan $2000,2011)$. Indeed, the experiment demonstrates the capacity for relatively minor behavioral differences to mediate the potential "heritability" of artifacts in cultural systems, which is a specific quantitative property possessed by artifact populations that can be conceived of as a ratio of the within-assemblage artifactual variation to the within-population behavioral variation of their makers that is directly influencing artifactual variability (Lycett 2016; Lycett et al. 2016). As 
shown elsewhere, this ratio is important since it is ultimately this variable that determines how quickly an artifactual tradition may respond to cultural evolutionary pressures of selection or drift in absolute terms (Lycett 2016; Lycett et al. 2016). Our experiments highlight how even subtle behavioral differences between individual artifact manufacturers can quantifiably influence this variable as a result of their effects at the artifactual level. Put simply, the type of small-scale artifactual differences we simulate here as a result of relatively small-scale behavioral manipulations, could potentially be the proximate source of more long-term and salient patterns of variation in archaeological datasets as a direct result of this proximate, behavioral basis.

One further implication of our experiment is that it speaks to the character of "cultural" variability that may be recorded in archaeological data. "Culture" can be defined straightforwardly as the socially learned behavioral differences in traditions, customs, and so forth that vary between different communities and subcommunities of individuals (Boyd and Richerson 1985); or, what might also be thought of as socially learned "ways of doing things" that differ between communities (McGrew 2004). It is certainly correct that culturally instilled attitudes toward shape might result in patterned differences between the shapes of artifacts of different artifact manufacturers (i.e., lead to visibly distinct material "traditions"). As one commentator put it, culturally learned concepts and biases might thus lead artifact manufacturers to think that "it should be just like this" as opposed to looking like that (Gowlett 2010:300). Asking a group of school children in the US to draw a "football" and then repeating the same task with a group of school children from Europe or Brazil, for instance, would quickly reveal the strength of such a process. However, archaeology has long had a tendency to think that measurable differences between artifacts are "cultural" only because there was an "intent" for artifact manufacturers to make their products this way as opposed to that way, or as a result of some specific "mental template" held in the minds of artifact producers (e.g., Deetz 1967:45). Even more forcefully, such differences might then be taken to mean that these are deliberate expressions of socio-cultural identity. Our experiment, however, whereupon all participants were motivated to copy the same "target" form, resulted in statistically patterned shape differences only because of a single behavioral difference in the two populations of artifact manufacturers, not because of any "intention" to deliberately produce systematically different artifactual forms. Our experiment, therefore, simulates what may happen in cases where two distinct "recipe" traditions of producing 
the same class of artifact-perhaps even only involving relatively small differences between communities in just a single behavioral "ingredient"-lead to empirically measurable differences at the artifactual level. In other words, the results of this experiment imply that when statistically significant differences are evident between two assemblages of arrowheads, pot forms, handaxes or any other form of artifact, the "cultural" difference lies not necessarily in a difference of direct intent to produce such a statistical shape difference, but in the socially learned behavioral manufacturing "ingredient" that proximately underlies such shape differences. These points echo certain statements made by Sackett (e.g., 1990) who argued on the basis of ethno-historical examples that what he termed "isochrestic style" might result from the behavioral factors underlying artifactual patterns as much as conscious "signaling." Our experiment highlights, however, just how minor such differences in behavioral tradition may need to be in order to produce empirically measurable differences between sets of artifacts separated by time or space. As we have highlighted, we certainly do not deny the role of culturally instilled attitudes toward shape ("functionally" related or otherwise) being a powerful force in the production of distinct traditions of artifacts. However, this experiment shows that empirically identifiable "cultural" differences may emerge at the artifactual-assemblage level simply as a result of subtle differences in manufacturing tradition (i.e., at the socially learned "recipe" level).

\section{Conclusion}

The results of our experiment highlight that an important potential engine of change, ultimately measurable in shape variability of artifacts over time and space, is relatively minor behavioral differences in single "input" variables of behavioral "recipes." In particular, small-scale behavioral differences may result in subtle but statistically patterned differences in artifactual form that is of microevolutionary relevance; that is, in terms of potential consequences for long-term and more profound differences in artifactual variability at a macroevolutionary scale. Measurable "cultural" differences between artifacts should be conceptualized not only potentially emerging as the result of culturally instilled attitudes toward the "correct" form of artifacts, but also potentially as the result of smaller-scale differences in behavioral ("ingredient") factors that comprise only relatively small components of socially learned artifactual recipes. The controlled and "artificial" character of our experiment aided in isolating and highlighting the role of a specific, discrete behavioral variable, which was ultimately measureable empirically at the assemblage level. Accordingly, 
these experiments further highlight the value of experiments involving "model artifacts," giving insights into small-scale factors that are as important to archaeology as those for which "model organisms" in biology are used for understanding fundamental, small-scale factors and processes, which are of major and profound consequence when exaggerated and extrapolated over larger and more long-term scales. 
Ethical statement:

The authors declare that they have no conflict of interest.

\section{References}

Ashburner, M., \& Novitski, E. (Eds.) (1976). The genetics and biology of Drosophila, Vol. 1 . New York: Academic Press.

Ashburner, M., Golic, K., \& Hawley, R.S. (2005). Drosophila: A laboratory handbook, 2 nd ed. New York: Cold Spring Harbor Laboratory Press.

Bataillon, T., Joyce, P., \& Sniegowski, P. (2013). As it happens: Current directions in experimental evolution. Biology letters, 9, 20120945.

Bleed, P. (1986). The optimal design of hunting weapons: maintainability or reliability? American Antiquity, 51, 737-747.

Bookstein, F.L. (1989). "Size and shape": A comment on semantics. Systematic Zoology, 38, 173-180.

Boyd, R., \& Richerson, P.J. (1985). Culture and the evolutionary process. Chicago: University of Chicago Press.

Buchanan, B., \& Hamilton, M.J. (2009). A formal test of the origin of variation in North American Early Paleoindian projectile points. American Antiquity, 74, 279-298.

Clarke, D.L. (1968). Analytical archaeology. London: Methuen.

Deetz, J. (1967). Invitation to archaeology. Garden City, New York: The Natural History Press.

Edwards, S.W. (2001). A modern knapper's assessment of the technical skills of the Late Acheulean biface workers at Kalambo Falls. In J.D. Clark (Ed.), Kalambo Falls Prehistoric Site, Vol. 3 (pp. 605-611). Cambridge: Cambridge University Press.

Eerkens, J.W. (2000). Practice effects makes within 5\% of perfect: Visual perception, motor skills, and memory in artifact variation. Current Anthropology, 41, 663-668.

Eerkens, J.W., \& Lipo, C.P. (2005). Cultural transmission, copying errors, and the generation of variation in material culture and the archaeological record. Journal of Anthropological Archaeology, 24, 316-334.

Eerkens, J.W., \& Lipo, C.P. (2007). Cultural transmission theory and the archaeological record: providing context to understanding variation and temporal changes in material culture. Journal of Anthropological Archaeology, 24, 316-334. 
Eren, M.I., Briggs, B., \& O’Brien, M.J. (2015). Social learning and technological evolution during the Clovis colonization of the New World. Journal of Human Evolution, 80, 159-170.

Falsetti, A.B., Jungers, W.L., \& Cole, T.M. (1993). Morphometrics of the Callitrichid forelimb: A case study in size and shape. International Journal of Primatology, 14, 551-572.

Futuyma, D. J., \& Bennett, A.F. (2009). The importance of experimental studies in evolutionary biology. In T. Garland \& M.R. Rose (Eds.), Experimental evolution: Concepts, methods, and applications (pp. 15-30). Berkeley: University of California Press.

Garland, T., \& Rose, M.R. (Eds.) (2009). Experimental evolution: Concepts, methods, and applications. Berkeley: University of California Press.

Ghiselin, M.T. (1969). The triumph of the Darwinian method. Berkeley: University of California Press.

Gowlett, J. A. J. (2006). The elements of design form in Acheulian bifaces: Modes, modalities, rules and language. In N. Goren-Inbar \& G. Sharon (Eds.), Axe age: Acheulian tool-making from quarry to discard (pp. 203-221). London: Equinox.

Gowlett, J. A. J. (2010). The future of lithic analysis in Palaeolithic archaeology: A view from the Old World. In S. J. Lycett \& P. R. Chauhan (Eds.), New perspectives on old stones: Analytical approaches to Palaeolithic technologies (pp. 295-309). New York: Springer.

Greenspan, R. J. (2004). Fly pushing: The theory and practice of Drosophila genetics, 2 nd ed. New York: Cold Springer Harbor Laboratory Press.

Hamilton, M. J., \& Buchanan, B. (2009). The accumulation of stochastic copying errors causes drift in culturally transmitted technologies: Quantifying Clovis evolutionary dynamics. Journal of Anthropological Archaeology, 28, 55-69.

Halpern, D.F. (2000). Sex differences in cognitive abilities, 3rd ed. Mahwah, New Jersey: Lawrence Erlbaum Associates.

Hammer, Ø., Harper, D.A.T., \& Ryan, P. (2001). PAST: Paleontological statistics software package for education and data Analysis. Palaeontologia Electronica, 4, 1-9.

Hampton, O.W. (1999). Culture of stone: Sacred and profane uses of stone among the Dani. Texas A\&M, College Station.

Heyes, C.M. (1994). Social learning in animals: Categories and mechanisms. Biological Reviews, 69, 207-231.

Jordan, P. (2015). Technology as a human social tradition: Cultural transmission among huntergatherers. Berkeley: University of California Press. 
Jungers, W.L., Falsetti, A.B., \& Wall, C.E. (1995). Shape, relative size, and size-adjustments in morphometrics. Yearbook of Physical Anthropology, 38, 137-161.

Kempe, M., Lycett, S.J., \& Mesoudi, A. (2012). An experimental test of the accumulated copying error model of cultural mutation for Acheulean handaxe size. PLoS ONE, 7, e48333.

Krause, R. (1985). The clay sleeps: An ethnoarchaeological study of three African potters. Tuscaloosa: University of Alabama Press.

Linn, M.C., \& Petersen, A.C. (1986). A meta-analysis of gender differences in spatial ability. In J. Shibley Hyde \& M. C. Linn (Eds.), The psychology of gender: Advances through metaanalysis (pp. 67-101). Baltimore, Maryland: The Johns Hopkins University Press.

Lycett, S.J. (2008). Acheulean variation and selection: Does handaxe symmetry fit neutral expectations? Journal of Archaeological Science, 35, 2640-2648.

Lycett, S.J. (2009). Quantifying transitions: morphometric approaches to Palaeolithic variability and technological change. In M. Camps \& P. R. Chauhan (Eds.), Sourcebook of Palaeolithic transitions: Methods, theories, and interpretations (pp. 79-92). New York: Springer.

Lycett, S.J. (2015). Cultural evolutionary approaches to artifact variation over time and space: basis, progress, and prospects. Journal of Archaeological Science, 56, 21-31.

Lycett, S.J. (2016). The importance of a "quantitative genetic" approach to the evolution of artifact morphological traits. In L. Mendoza-Straffon (Ed.), Cultural phylogenetics: Concepts and applications in archaeology and anthropology (in press). New York: Springer Press.

Lycett, S.J., \& von Cramon-Taubadel, N. (2015). Toward a "quantitative genetic" approach to lithic variation. Journal of Archaeological Method and Theory, 22, 646-675.

Lycett, S.J., von Cramon-Taubadel, N., \& Foley, R.A. (2006). A crossbeam co-ordinate caliper for the morphometric analysis of lithic nuclei: A description, test and empirical examples of an application. Journal of Archaeological Science, 33, 847-861.

Lycett, S.J., Schillinger, K., Kempe, M., \& Mesoudi, A. (2015). Learning in the Acheulean: Insights from experiments using handaxe form as a "model organism". In A. Mesoudi \& K. Aoki (Eds.), Learning strategies and cultural evolution during the Paleolithic (pp.155-166). New York, Springer.

Lycett, S.J., Schillinger, K., Eren, M.I., von Cramon-Taubadel, N., \& Mesoudi, A. (2016). Factors affecting Acheulean handaxe variation: Experimental insights, microevolutionary processes, and macroevolutionary outcomes. Quaternary International, http://dx.doi.org/10.1016/j.quaint.2015.08.021 
Lyman, R.L., VanPool, T.L., \& O’Brien, M.J. (2009). The diversity of North American projectile-point types, before and after the bow and arrow. Journal of Anthropological Archaeology, 28, 1-13.

Mahius, M.C. (1993). Pottery techniques in India: technical variants and social choice. In P. Lemonnier (Ed.), Technological choices: transformation in material cultures since the Neolithic. London: Routledge.

McGrew, W.C. (2004). The cultured chimpanzee: Reflections on cultural primatology. Cambridge: Cambridge University Press.

Meltzer, D. J. (1981). A study of style and function in a class of tools. Journal of Field Archaeology, 8, 313-326.

Mesoudi, A. (2011). Cultural evolution: How Darwinian theory can explain human culture and synthesize the social sciences. Chicago: University of Chicago Press.

Mesoudi, A., \& O’Brien, M.J. (2008). The cultural transmission of Great Basin projectile point technology I: An experimental simulation. American Antiquity, 73, 3-28.

Mueller, L. D. (2009). Fitness, demography, and population dynamics in laboratory experiments. In T. Garland \& M. R. Rose (Eds.), Experimental evolution: Concepts, methods, and applications (pp. 197-216). Berkeley: University of California Press.

Neiman, F.D. (1995). Stylistic variation in evolutionary perspective: Inferences from decorative diversity and interassemblage distance in Illinois Woodland ceramic assemblages. American Antiquity, 60, 7-36.

O’Brien, M.J., \& Lyman, R.L. (2000). Applying evolutionary archaeology: A systematic approach. New York: Kluwer Academic/Plenum.

O’Brien, M.J., Holland, T.D., Hoard, R.J., \& Fox, G.L. (1994). Evolutionary implications of design and performance characteristics of prehistoric pottery. Journal of Archaeological Method and Theory, 1, 259-304.

O'Brien, M.J., Lyman, R.L., Mesoudi, A., \& VanPool, T.L. (2010). Cultural traits as points of analysis. Philosophical Transactions of the Royal Society B, 365, 3797-3806.

Orton, C., Tyers, P., \& Vince, A. (1993). Pottery in archaeology. Cambridge: Cambridge University Press.

Reid, J.J., Schiffer, M.B., \& Rathe, W.L. (1975). Behavioral archaeology: Four strategies. American Anthropologist, 77, 864-869.

Rice, P. M. (1987). Pottery analysis: A source book. University of Chicago Press. 
Robert, M., \& Chevrier, E. (2003). Does men's advantage in mental rotation persist when real three-dimensional objects are either felt or seen? Memory \& Cognition, 31, 1136-1145.

Roche, H. (2005.) From simple flaking to shaping: Stone-knapping evolution among early hominins. In V. Roux \& B. Bril (Eds.), Stone knapping: The necessary conditions for a uniquely hominin behavior (pp. 35-48). Cambridge: Mc-Donald Institute Monographs.

Rogers, D.S., Feldman, M.W., \& Ehrlich, P.R. (2009). Inferring population histories using cultural data. Proceedings of the Royal Society of London B, 276, 3835-3843.

Rohlf, F.J. (2010). TpsDig2 v2.16. Stony Brook, N.Y., Department of Ecology and Evolution, State University of New York. Electronic document. http://life.bio.sunysb.edu/morph/. Accessed March 4, 2015.

Sackett, J.R. (1990). Style and ethnicity in archaeology: the case for isochrestism. In M.W. Conkey \& C.A. Hastorf (Eds.), The uses of style in archaeology (pp. 32-43). Cambridge: Cambridge University Press.

Schiffer, M.B., \& Skibo, J.M. (1987). Theory and experiment in the study of technological change. Current Anthropology, 28, 595-622.

Schiffer, M.B., \& Skibo, J.M. (1997). The explanation of artifact variability. American Antiquity, $62,27-50$.

Schillinger, K., Mesoudi, A., \& Lycett, S.J. (2014a). Copying error and the cultural evolution of "additive" versus "reductive" material traditions: An experimental assessment. American Antiquity, 79, 128-143.

Schillinger, K., Mesoudi, A., \& Lycett, S.J. (2014b). Considering the role of time budgets on copy-error rates in material culture traditions: An experimental assessment. PloS One, 9, e97157.

Schillinger, K., Mesoudi, A., \& Lycett, S.J. (2015). The impact of imitative versus emulative learning mechanisms on artifactual variation: Implications for the evolution of material culture. Evolution and Human Behavior, 36, 446-455.

Shennan, S. (2000). Population, culture history, and the dynamics of culture change. Current Anthropology, 41, 811-835.

Shennan, S. (2011). Descent with modification and the archaeological record. Philosophical Transactions of the Royal Society B, 366, 1070-1079.

Shepard, A.O. (1965). Ceramics for the archaeologist. Publication No. 609. Washington, D.C: Carnegie Institution of Washington.

Skibo, J. M., \& Schiffer, M.B. (1995). The clay cooking pot: An exploration 
of women's technology. In J.M. Skibo, W. H. Walker, \& A.E. Nielsen (Eds.), Expanding Archaeology (pp. 80-91). Salt Lake City: University of Utah Press.

Skibo, J.M., \& Schiffer, M.B. (2001). Understanding artifact variability and change: A behavioral framework. In M.B. Schiffer (Ed.), Anthropological perspectives on technology (pp. 139-149). Albuquerque: University of New Mexico Press.

Sokal, R., \& Rohlf, F.J. (1995). Biometry, 3rd ed. New York: W.H. Freeman.

Smallwood, A.M. (2012). Clovis technology and settlement in the American Southeast: Using biface analysis to evaluate dispersal models. American Antiquity, 77, 689-713.

VanPool, T.L. (2001). Style, function, and variation: Identifying the evolutionary importance of traits in the archaeological record. In T.D. Hunt \& G.F.M. Rakita (Eds.), Style and function: Conceptual issues in evolutionary archaeology (pp. 119-140). Westport, Connecticut: Bergin and Garvey.

VanPool, T.L., Palmer, C.T., VanPool, C.S. (2008). Horned serpents, tradition, and the tapestry of culture. In M.J. O'Brien (Ed.), Cultural transmission and archaeology: Issues and case studies (pp. 77-90). Washington, D.C.: Society for American Archaeology Press.

Voyer, D., Voyer, S., \& Bryden, M.P. (1995). Magnitude of sex differences in spatial abilities: A meta-analysis and consideration of critical variables. Psychological Bulletin, 117, 250-270.

Wendrich, W. (1999). The world according to basketry: An ethno-archaeological interpretation of basketry production in Egypt. Leiden: Research School of Asian, African and Amerindian Studies, Universiteit Leiden.

Wynn, T.G., Tierson, F.D. \& Palmer, C.T. (1996). Evolution of sex differences in spatial cognition. Yearbook of Physical Anthropology, 39, 11-42. 


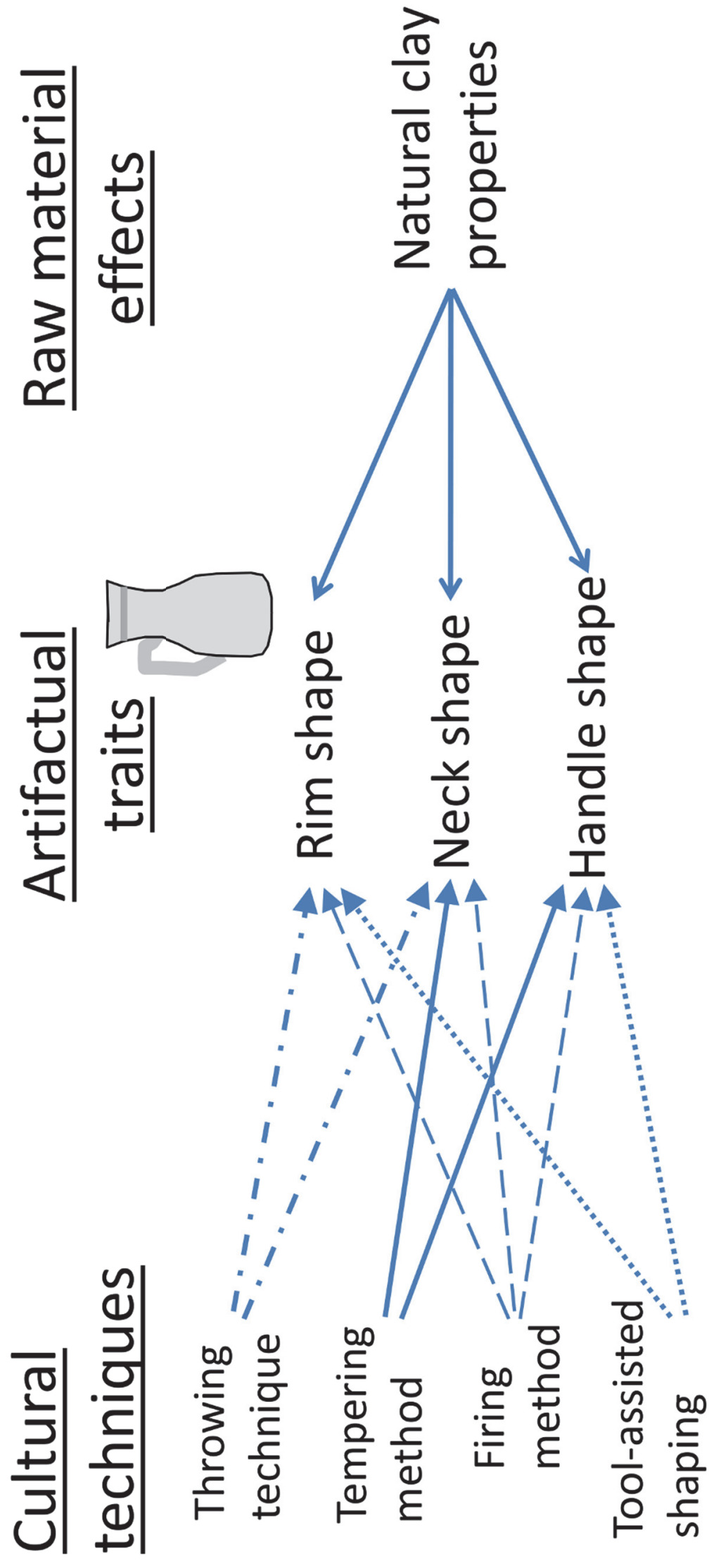

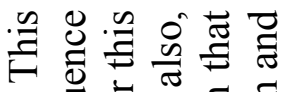

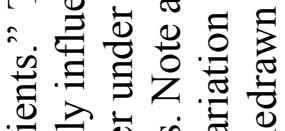

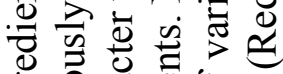

bo

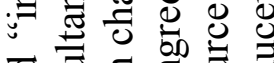

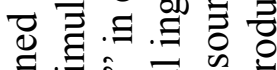

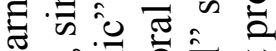

ऽ

$\lambda ن$

.

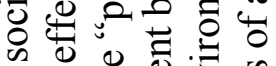

可娄

䜦

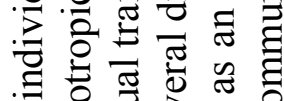

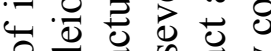

은

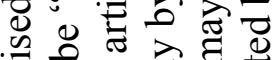

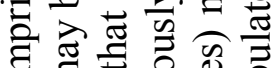

0 品

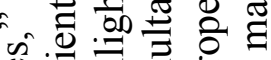

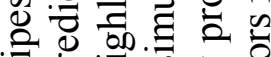

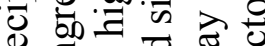

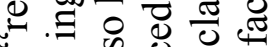

उ

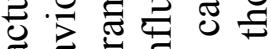

政

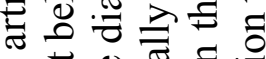

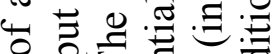

금

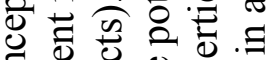

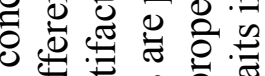

氜兘总

Ð

प등 항

ప

.0.0. 웡

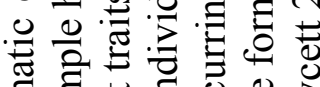

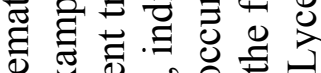

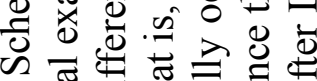
$\because \overline{0}=$

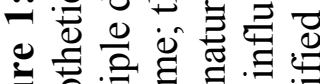

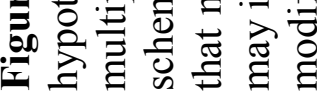




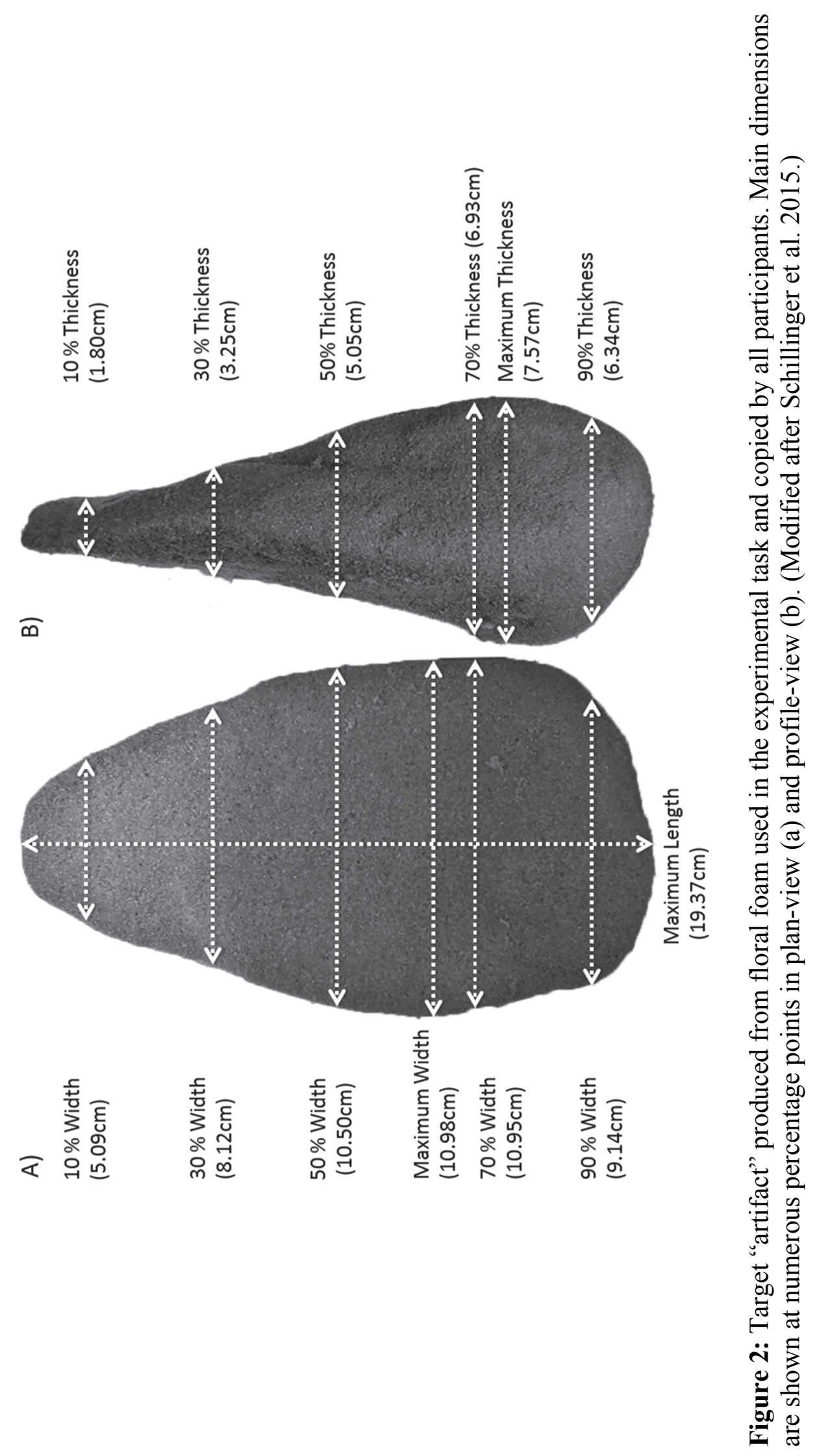


$N$
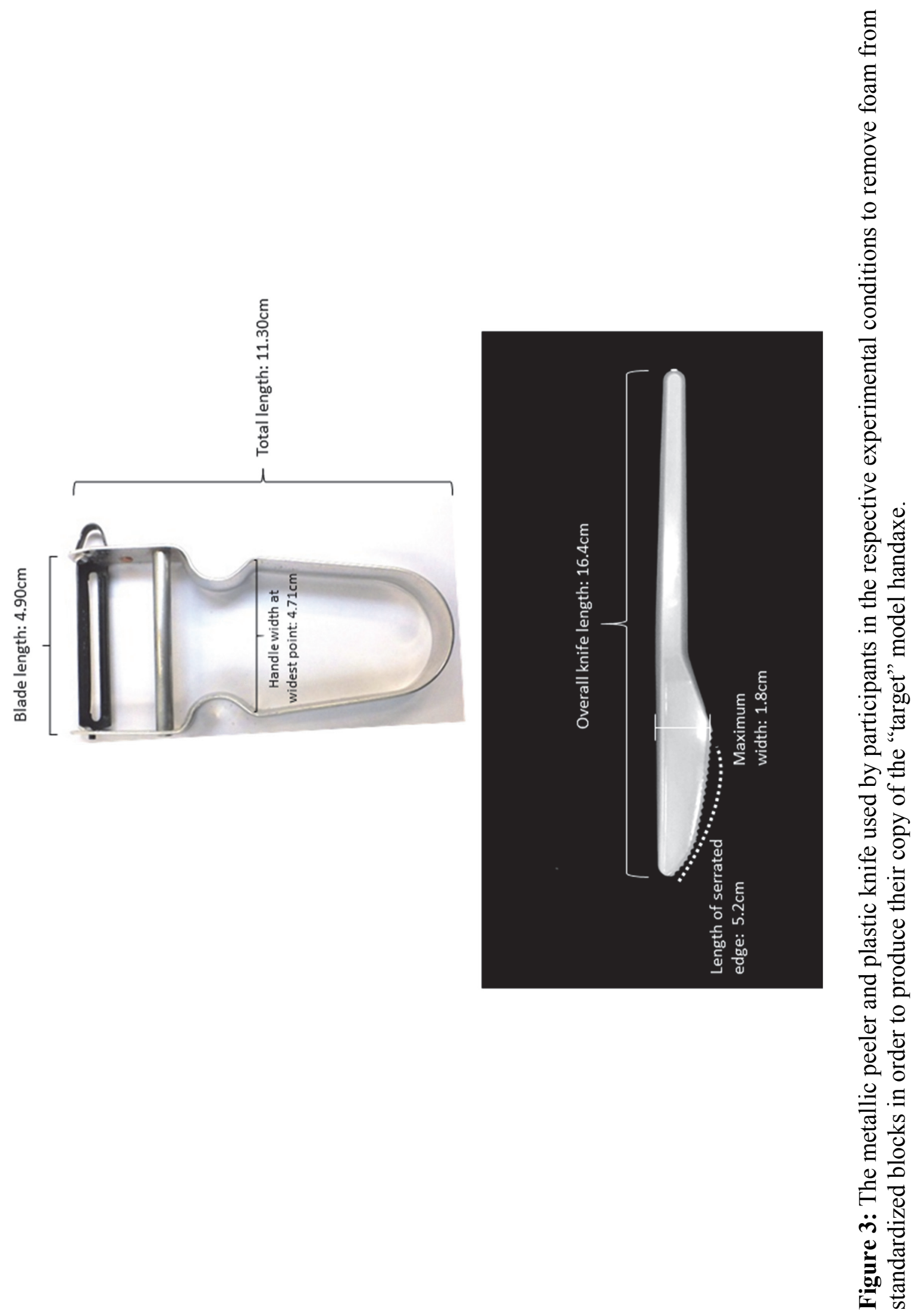


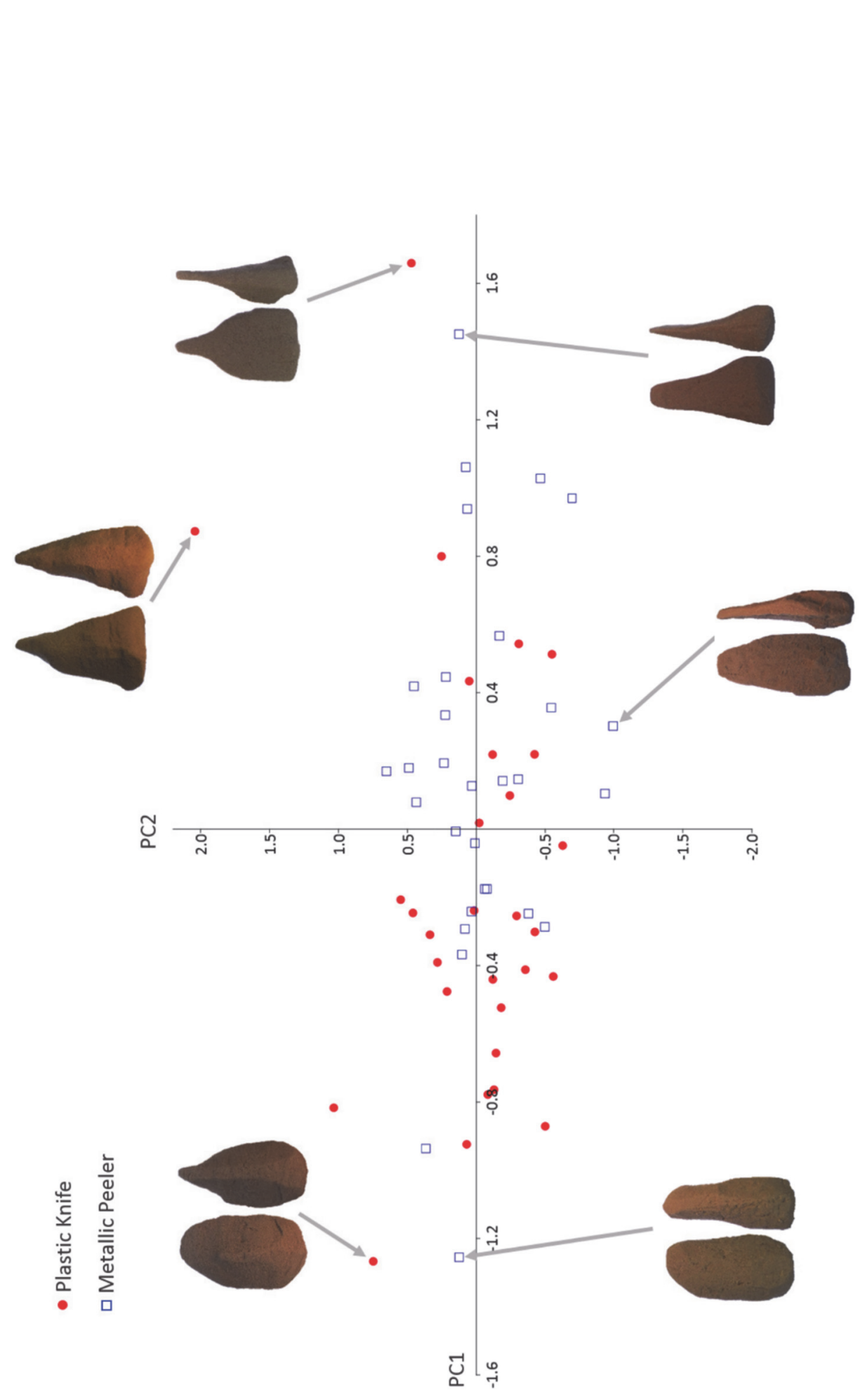

๙ ฮี 뭏

ते 3 弪

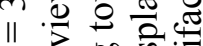

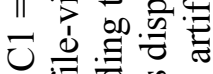

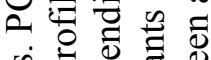

வे

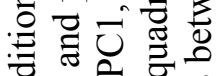

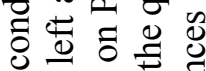

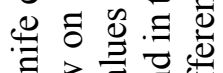

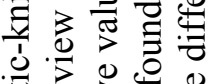

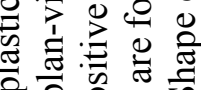

를 क ज

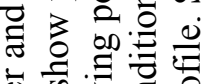

过 䨌

줄

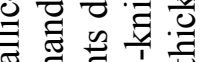

元要选

ఏ

采

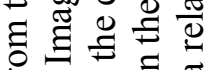

일.

苍.

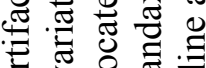

齐。寻

फ

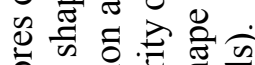

㶽

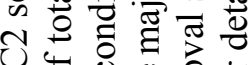

Uफ

후워

可 卷

ठ

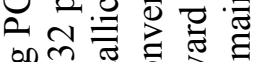

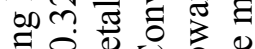

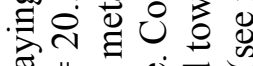

त् $\|$ o

包记志可

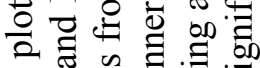

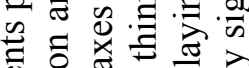

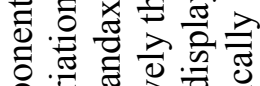

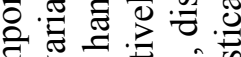

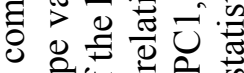

过范

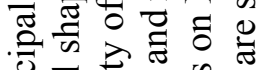

:

ㄴ.

$\ddot{7} 0$ घ

氙《号. ठ

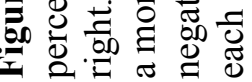

